\title{
Drug-specific in vitro release of IFN-gamma in patients with delayed cutaneuos drug hypersensitivity reactions
}

\author{
Grzegorz Porebski ${ }^{1 *}$, Magdalena Bosak² \\ From EAACI Skin Allergy Meeting 2014 \\ Krakow, Poland. 18-20 September 2014
}

\section{Background}

One of the challenges for management of drug hypersensitivity reactions (DHR) is to detect a culprit drug. Although measures of interferon IFN-gamma production by patients' drug-specific $\mathrm{T}$ cells have been the widely utilized cytokine assay, a systematic comparison of different methods has not yet been reported.

\section{Method}

A total of 16 patients with clinically well-established maculopapular exanthema due to antiepileptic drugs hypersensitivity in remission state and 15 drug-exposed control donors without DHR were included to the study. Peripheral blood mononuclear cells of investigated individuals were isolated and cultured under defined conditions with drugs. IFN-gamma production was measured with electrochemiluminescence array assay and ELISA (cytokine level in cell culture supernatant), ELISpot (cytokine secreting cells), flow cytometry (intracellular staining in $\mathrm{CD} 3+\mathrm{CD} 4+$ cells).

\section{Results}

IFN-gamma production could be demonstrated in 13 of 16 patients using electrochemiluminescence assay (sensitivity $81 \%$ ), in 8 of 16 patients using ELISA (sensitivity 50\%), in 6 of 16 and 7 of 16 patients using ELISpot (sensitivity $46 \%$ ) and flow cytometry (sensitivity 57\%), respectively. The sensitivity of combined measurements of drug-specific IFN-gamma by ELISpot, ELISA and flow cytometry achieved $88 \%$. Healthy controls showed negative drug-specific IFN-gamma production in contrast to individuals with a known sensitivity in all tested read-out systems.

The assays demonstrated a test specificity of $100 \%$ (electrochemiluminescence), 93\% (ELISA), 100\% (ELISpot) and $100 \%$ (flow cytometry).

\section{Conclusion}

Analysis of drug-specific IFN-gamma production by means of different assays proved a useful and reliable approach for the in vitro detection of drug hypersensitivities in the investigated population. Electrochemiluminescence array assay offers distinct advantage over the other tested assays, including a greater sensitivity, but its availability is limited because of the costs. Also combining different assays may be a feasible approach to identify the causative drug of DHR.

\section{Authors' details}

'Jagiellonian University, Dep. of Clinical and Environmental Allergology,

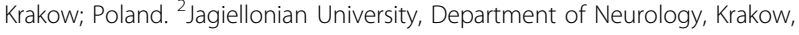
Poland.

Published: 11 March 2015

doi:10.1186/2045-7022-5-S1-O3

Cite this article as: Porebski and Bosak: Drug-specific in vitro release of IFN-gamma in patients with delayed cutaneuos drug hypersensitivity reactions. Clinical and Translational Allergy 2015 5(Suppl 1):O3. 